\section{Thermal decomposition of solids}

RAJESHWAR et al. ${ }^{1}$ are probably the first to use differential scanning calorimetry (DSC) to study certain solid-state reactions in the presence of high electric fields within $100-400 \mathrm{kV} \mathrm{m}^{-1}$. Shifts in temperature of the DSC peaks, changes of areas under individual peaks, or both were observed. The authors regarded these phenomena as kinetic effects of the field on thermal decomposition, and with this assumption discussed briefly the various mechanisms that may be responsible.

We would like to make two comments. First, we suggest that some of the reactions examined are not thermal decompositions enhanced by the field, but electrically-induced degradations accelerated by the rising temperature. Application of low fields such as $5-25 \mathrm{kV} \mathrm{m}^{-1}$ is known to increase the rate of some thermal decomposition reactions (see, for example, refs 2-4). However, when high fields are involved charge carrier injection may occur and a novel type of 'electrochemical' mechanism may operate in ionic metallic compounds including sodium azide ${ }^{5}$. The electric fields applied in the experiments on $\mathrm{NaN}_{3}$ have not been specified $^{1}$ but, if they are of the order of $10^{5} \mathrm{~V} \mathrm{~m}^{-1}$ as reported for other substances, they would have been intense enough $^{6}$ to cause partial chemical changes in a single crystal at room temperature by this mechanism. Rajeshwar et al. ${ }^{1}$ have studied samples in powder form at elevated temperatures; the two differences in experimental conditions may be mutually compensating. In the case of $\mathrm{AgN}_{3}$, the critical field for compacted powder is an order of magnitude greater than that for a single crystal, but the induction period for the onset of electronhole injection decreases when the temperature is raised ${ }^{7,8}$.

The second point concerns the 'pronounced' and 'drastic' increases of exothermicity observed during the decompositions of $\mathrm{NaN}_{3}$ and $\mathrm{KMnO}_{4}$. The former decomposes according to the equation

$$
2 \mathrm{NaN}_{3} \rightarrow 2 \mathrm{Na}+3 \mathrm{~N}_{2}
$$

The extent of the above reaction, although dependent on the crystal size and temperature ${ }^{9}$, should reach completion in the experiments under discussion since the temperature was raised continuously to $623 \mathrm{~K}$ or higher. For metallic azides excepting the alkaline-earth compounds, the measured heats of reactions, $Q$, closely approach the corresponding heats of formation, $\Delta H_{t}$. It would be interesting to compare the $Q$ value obtained by the authors with the $\Delta H_{f}$ of $\mathrm{NaN}_{3}$ which is $322 \mathrm{~kJ} \mathrm{~kg}^{-1}$. If $Q$ (with electric field) exceeds $\Delta H_{\mathrm{f}}$, then we suspect that joule heating ${ }^{6}$ is responsible for their difference. The decomposition of $\mathrm{KMnO}_{4}$ is complex in nature. From its DSC curves ${ }^{1}$ we estimate $Q$ at 0 and $200 \mathrm{kV} \mathrm{m}^{-1}$ to be $\sim 5$ and $300 \mathrm{~kJ} \mathrm{~kg}^{-1}$ respectively. In view of this drastic change in $Q$, we suggest that chemical characterization of the residues be carried out to clarify both the extent of decomposition and whether the reaction products are the same in both cases.

We also feel that experiments should be performed with the sample placed in

Matters Arising
Matters Arising is meant as a vehicle
for comment and discussion about
papers that appear in Nature. The
originator of a Matters Arising
contribution should initially send his
manuscript to the author of the ori-
ginal paper and both parties should,
wherever possible, agree on what is to
be submitted. Neither contribution
nor reply (if one is necessary) should
be longer than 500 words and the
briefest of replies, to the effect that a
point is taken, should be considered.

an electric field applied through insulating electrodes, as has been done for silver azide $^{5}$. This arrangement will prevent charge carrier injection and eliminate joule heating. Any kinetic or thermochemical effects found in such conditions can be truly attributed to the influence of electric field on thermal decomposition.

T. B. TANG
V. KRISHNA MOHAN
Department of Physics,
University of Cambridge,
Madingley Road,
Cambridge CB3 OHE, UK

1. Rajeshwar, K., Rosenvold, R. \& Du Bow, J. Nature 301, 48-49 (1983).

2. Kabanov, A. A. Russ, chem. Rev. 40, 953-963 (1971)

3. Pai Verneker, V., Sood, R. K. \& Krishna Mohan, V. Ind. J. Chem. 13, 908-912 (1975).

4. Pai Verneker, V., Kishore, K. \& Sunitha, M. R. J. Solid St. Chem. 26, 203-206 (1978)

5. Tang, T. B. \& Chaudhri, M. M. Nature 282, 54-51 (1979).

6. Tang, T. B. thesis, Cambridge Univ. (1979).

7. Robinson, C. J. thesis, Cambridge Univ. (1981).

8. Robinson, C. J. \& Chaudhri, M. M. Proc. 1st Inst. Conf. Conduction and Breakdown in Solid Dielectrics, Toulouse (in the press).

9. Walker, R. F., Gane, N. \& Bowden, F. P. Proc. R. Soc A294, 417-436 (1966).

\section{Factors underlying falcon grating acuity}

IN investigating falcon visual sensitivity to grating contrast ${ }^{1}$, the contrast sensitivity functions of falcon and human observers were determined using stationary and phase changing gratings with a luminance of $40 \mathrm{~cd} \mathrm{~m}^{-2}$. The study provides a qualitative comparison of falconiforme and human visual performance at that particular luminance, but the data do not warrant the quantitative analysis given for the possible factors underlying spatial acuity in the falconiforme deep fovea.

The extrapolated sensitivity functions show equal visual acuities (expressed in terms of cutoff frequency) for falcon and man. The author argues that the falcon shows an 'extraordinarily high' visual performance because the anatomical proportions of the subjects' eyes indicate a maximum falcon acuity three-quarters that of man. Since the observed human cutoff frequency was 40 cycles $\mathrm{deg}^{-1}$, the expected proportional falcon cutoff frequency would be 30 cycles deg $^{-1}$ rather than the observed value of 40 cycles$\mathrm{deg}^{-1}$. To account for this boost in acuity the theory that the spherical pit of the deep fovea acts as the divergent element in a telephoto lens system ${ }^{2}$ is invoked. In this case, the spherical pit needs to provide an image magnification factor of 1.33. We feel that it is not necessary, nor in this case applicable, to suggest such a theory to explain falcon acuity when measured under the relatively low luminance of $40 \mathrm{~cd} \mathrm{~m}^{-2}$.

To assess whether the falcon's acuity is 'extraordinarily high' or not, its behavioural acuity performance needs to be related to the potential anatomical performance of its own eye, rather than the behavioural performance of another species. The anatomical cutoff frequency of the falcon eye can be defined by the equation and data given in Hirsch's paper $^{1}$ :

$$
\nu=\frac{F}{d_{\mathrm{cc}} \sqrt{3} \times 57.3} \times m
$$

where $\nu$ is the cutoff frequency in cycles $\operatorname{deg}^{-1}, F$ is the falcon focal length of $9.1 \mathrm{~mm}, d_{\mathrm{cc}}$ represents photoreceptor centre-to-centre spacing of $2 \mu \mathrm{m}$, and $m$ is the foveal magnification factor. Assuming no magnification, $m$ is equal to 1 . According to this equation the dimensions of the falcon eye allow for a cutoff frequency of 46 cycles deg $^{-1}$, which is above the behaviourally measured acuity of 40 cycles deg $^{-1}$. It appears that a measured acuity of 40 cycles deg $^{-1}$ is not 'extraordinarily high'. Note that the last measured response for stationary gratings was obtained at $\sim 25$ cycles $\mathrm{deg}^{-1}$ The 